\title{
First Chromosome Analysis and Localization of the Nucleolar Organizer Region of Land Snail, Sarika resplendens (Stylommatophora, Ariophantidae) in Thailand
}

\author{
Wilailuk Khrueanet ${ }^{1}$, Weerayuth Supiwong ${ }^{2}$, Chanidaporn Tumpeesuwan ${ }^{3}$, \\ Sakboworn Tumpeesuwan ${ }^{3}$, Krit Pinthong ${ }^{4}$, and Alongklod Tanomtong ${ }^{2 *}$ \\ ${ }^{1}$ School of Science and Technology, Khon Kaen University, \\ Nong Khai Campus, Muang, Nong Khai 43000, Thailand \\ ${ }^{2}$ Applied Taxonomic Research Center (ATRC), Department of Biology, Faculty of Science, \\ Khon Kaen University, Muang, Khon Kaen 40002, Thailand \\ ${ }^{3}$ Department of Biology, Faculty of Science, Mahasarakham University, \\ Kantarawichai, Maha Sarakham 44150, Thailand \\ ${ }^{4}$ Biology Program, Faculty of Science and Technology, Surindra Rajabhat University, \\ Muang, Surin 32000, Thailand
}

Received July 23, 2012; accepted February 25, 2013

\begin{abstract}
Summary We report the first chromosome analysis and localization of the nucleolar organizer region of the land snail Sarika resplendens (Philippi 1846) in Thailand. The mitotic and meiotic chromosome preparations were carried out by directly taking samples from the ovotestis. Conventional and Ag-NOR staining techniques were applied to stain the chromosomes. The results showed that the diploid chromosome number of $S$. resplendens is $2 n=66$ and the fundamental number (NF) is 132. The karyotype has the presence of six large metacentric, two large submetacentric, 26 medium metacentric, and 32 small metacentric chromosomes. After using the Ag-NOR banding technique, one pair of nucleolar organizer regions (NORs) was observed on the long arm subtelomeric region of chromosome pair 11. We found that during metaphase I, the homologous chromosomes show synapsis, which can be defined as the formation of 33 ring bivalents, and 33 haploid chromosomes at metaphase II as diploid species. The karyotype formula of $S$. resplendens could be deduced as:
\end{abstract}

(2n=66): $\mathrm{L}_{6}^{\mathrm{m}}+\mathrm{L}_{2}^{\mathrm{sm}}+\mathrm{M}_{26}^{\mathrm{m}}+\mathrm{S}_{32}^{\mathrm{m}}$

Key words Sarika resplendens, Karyotype, Chromosome, Nucleolar organizer region.

Thailand is a tropical country where the weather and environment are suitable for many organisms, resulting in high biodiversity. Wild animals are one group that show this biodiversity and are also important in ecology and the food chain. Land snails, freshwater snails, and slugs (Mollusca, Gastropoda, Pulmonata) include some 30,000-50,000 species (Solem 1984). Evolutionary inter-relationships within the Pulmonates remain largely unresolved as proved by several rearrangements of their systematic (Wade et al. 2001, Vitturi et al. 2005). The chromosome numbers of ariophantid snails have been reported by Patterson and Burch (1978). They reported that the haploid chromosome number $(n)$ ranged from $n=27$ to $n=32$, but the karyotype was not reported. Some species of the family Ariophantidae have been studied from a cytogenetic viewpoint, and most authors have reported a diploid chromosome number $(2 n)$ that ranged from $2 n=16$ to $2 n=66$ (Table 1). It is well known that banding techniques such as silver impregnation (Ag-NOR banding), C-banding, G-banding, and fluorochrome staining have been successfully used to obtain knowledge on genomic DNA complexity and organization in animals (Sumner 1990).

*Corresponding author, e-mail: tanomtong@hotmail.com

DOI:1.1508/cytologia.78.213 
Table 1. Review of cytogenetic reports of land snails in the family Ariophantidae (genera; Sarika, Cryptozona, Hemiplecta, Macrochlamys, Syama, Dyakia, Megaustenia, and Durgella).

\begin{tabular}{|c|c|c|c|c|c|}
\hline Species & $2 n$ & Karyotype formulas & NF & NOR banded & References \\
\hline Sa. resplendens & 66 & $\mathrm{~L}_{6}^{\mathrm{m}}+\mathrm{L}_{2}^{\mathrm{sm}}+\mathrm{M}_{26}^{\mathrm{m}}+\mathrm{S}_{32}^{\mathrm{m}}$ & 132 & 11 (STR) & Present study \\
\hline \multirow{2}{*}{ C. siamensis } & 66 & - & - & - & Dumrongrojwattana et al. (2007) \\
\hline & 16 & - & - & - & Vitturi et al. (1995) \\
\hline C. bisgrialis & 16 & $8 m+2 s m+2 s t+4 t$ & 28 & - & Mattayassook (1996) \\
\hline \multirow{2}{*}{ H. distincta } & 64 & - & - & - & Patterson and Burch (1978) \\
\hline & 66 & - & - & - & Dumrongrojwattana et al. (2007) \\
\hline \multirow[t]{2}{*}{ H. weinkauffiana } & 60 & $18 \mathrm{mac}+42 \mathrm{micro}$ & - & - & Mattayassook (1996) \\
\hline & 66 & - & - & - & Dumrongrojwattana et al. (2007) \\
\hline \multirow[t]{2}{*}{ Sa. asamurai } & 58 & - & - & - & Mattayassook (1996) \\
\hline & 66 & $58 \mathrm{~m}+8 \mathrm{sm}$ & - & - & Dumrongrojwattana et al. (2005) \\
\hline Ma. indica & 48 & $18 \mathrm{~m}+30 \mathrm{sm}$ & - & - & El Alfy (1994) \\
\hline Ma. splendens & 20 & $10 \mathrm{~m}+2 \mathrm{sm}+8 \mathrm{t}$ & 32 & - & Mattayassook (1996) \\
\hline \multirow[t]{3}{*}{ Sa. diadema } & 20 & $16 \mathrm{~m}+4 \mathrm{sm}$ & 40 & - & Mattayassook (1996) \\
\hline & 66 & $56 \mathrm{~m}+10 \mathrm{sm}$ & - & - & Dumrongrojwattana et al. (2005) \\
\hline & 66 & - & - & - & Dumrongrojwattana et al. (2007) \\
\hline \multirow[t]{3}{*}{ Dy. salangana } & 66 & - & - & - & Dumrongrojwattana et al. (2007) \\
\hline & $50-54$ & - & - & - & Mattayassook (1996) \\
\hline & $50-54$ & - & - & - & Vitturi et al. (1995) \\
\hline Me. siamensis & 66 & - & - & - & Dumrongrojwattana et al. (2007) \\
\hline Du. libas & 66 & - & - & - & Dumrongrojwattana et al. (2007) \\
\hline
\end{tabular}

Remarks: $2 n=$ diploid chromosome number, $\mathrm{NF}=$ fundamental number (number of chromosome arm), $\mathrm{m}=$ metacentric chromosome, $\mathrm{sm}=$ submetacentric chromsome, $\mathrm{a}=$ acrocentric chromosome, $\mathrm{st}=$ subtelocentric chromosome, $\mathrm{t}=$ telocentric chromosome, $\mathrm{STR}=$ on subtelomeric region, and $-=$ not available.

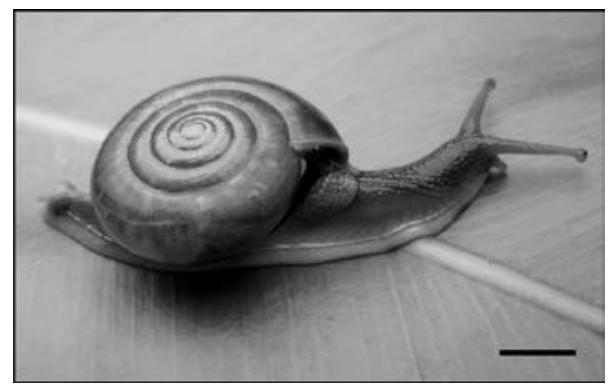

Fig. 1 General characteristics of the land snail, Sarika resplendens (Philippi 1846) in Thailand (Stylommatophora, Ariophantidae, Macrochlamydinae) (scale bar=1 cm).

The land snail Sarika resplendens (Philippi 1846) is member of the family Ariophantidae. According to the classification of Vaught (1989), this family comprises 69 genera, of which only 25 species in 13 genera have been reported from Thailand (Panha 1996, Hemmen and Hemmen 2001, Nabhitabhata 2009). S. resplendens belongs to the kingdom Animalia, phylum Mollusca, class Gastropoda, subclass Pulmonata, order Stylommatophora, superfamily Helicarionoidea, family Ariophantidae, and subfamily Macrochlamydinae. The general characteristics of $S$. resplendens are as follows: shell perforate, depressed, smooth, polished throughout, thin, pale, yellowish tawny, without longitudinal sculpture; spire very low, suture well impressed; whorls 7, convex, regularly increasing, with the last rounded at the periphery and convex, slightly flattened beneath, deeply impressed in the middle; aperture nearly vertical, broadly lunate; peristome thin, almost straight, basal margin faintly arcuate, columellar oblique, and briefly reflected above (Blanford and Austen 1908) (Fig. 1). 
A literature review of the cytogenetic studies of land snails in the family Ariophantidae was conducted, which includes works by Patterson and Burch (1978), Vitturi et al. (1995), Mattayassook (1996), El Alfy (1994) and Dumrongrojwattana et al. (2005, 2007). The objective of this study is to investigate the karyotype analysis and chromosomal characteristic of NORs in S. resplendens from Thailand by conventional staining and the Ag-NOR banding technique. This study expands the existing cytogenetic knowledge, and will be useful for further studies on taxonomy and evolutionary relationships. Furthermore, the basic information obtained will be useful for the conservation and chromosome evolution studies of these land snails.

Materials and methods

\section{Sample collection}

S. resplendens (four specimens) was obtained from Nakhon Ratchasima Province, Thailand. The snails were transferred to the laboratory and kept under standard conditions for three days prior to the experiments.

\section{Chromosome preparation}

Chromosomes were directly prepared in vivo (Kawano and Leme 1994) as follows. Colchicines solution was injected into the snails' intramuscular, and then they were left alone for $8 \mathrm{~h}$. Ovotestis samples were cut into small pieces, squashed, and then mixed with $0.075 \mathrm{M} \mathrm{KCl}$. After discarding all large tissue pieces, $15 \mathrm{ml}$ of the cell sediments were transferred to a centrifuge tube and incubated for $25-35 \mathrm{~min}$. The $\mathrm{KCl}$ was discarded from the supernatant after another centrifugation at 1,200 rpm for $8 \mathrm{~min}$. The cells were fixed by gradually adding $8 \mathrm{ml}$ of fresh cool fixative (3 methanol: 1 glacial acetic acid) before centrifuging again at 1,200 rpm for $8 \mathrm{~min}$, after which the supernatant was discarded. The fixation was repeated until the supernatant was clear, and the pellet was mixed with $1 \mathrm{ml}$ of the fixative. The mixture was micropipetted onto a clean, cold slide, and then an air-drying technique was applied.

\section{Chromosome staining}

Conventional staining was done using 20\% Giemsa's solution for $30 \mathrm{~min}$. Ag-NOR banding (Howell and Black 1980) was performed by adding two drops of 50\% silver nitrate and $2 \%$ gelatin on the slides. The slides were then sealed with cover glasses and incubated at $60^{\circ} \mathrm{C}$ for $5 \mathrm{~min}$. Next, the slides were soaked in distilled water until the cover glasses separated and were stained with $20 \%$ Giemsa's solution for $1 \mathrm{~min}$.

\section{Chromosome checks}

Chromosome counting was performed on mitotic metaphase cells under a light microscope. Ten clearly observable cells with well-spread chromosomes of each specimen were selected and photographed. The length of the short arm chromosome (Ls) and the length of long arm chromosome (Ll) were measured to calculate the length of the total arm chromosome ( $\mathrm{LT}, \mathrm{LT}=\mathrm{Ls}+\mathrm{Ll})$. The relative length (RL) and the centromeric index (CI) were estimated (Chaiyasut 1989). Chromosomes with CI $(\mathrm{q} / \mathrm{p}+\mathrm{q})$ between $0.50-0.59,0.60-0.69,0.70-0.89$, and $0.90-0.99$ are described as metacentric, submetacentric, acrocentric, and telocentric chromosomes, respectively. The fundamental number (number of chromosome arms, NF) was obtained by assigning a value of two to metacentric, submetacentric, and acrocentric chromosomes, and a value of one to telocentric chromosomes. All parameters were used in karyotyping and idiograming. 

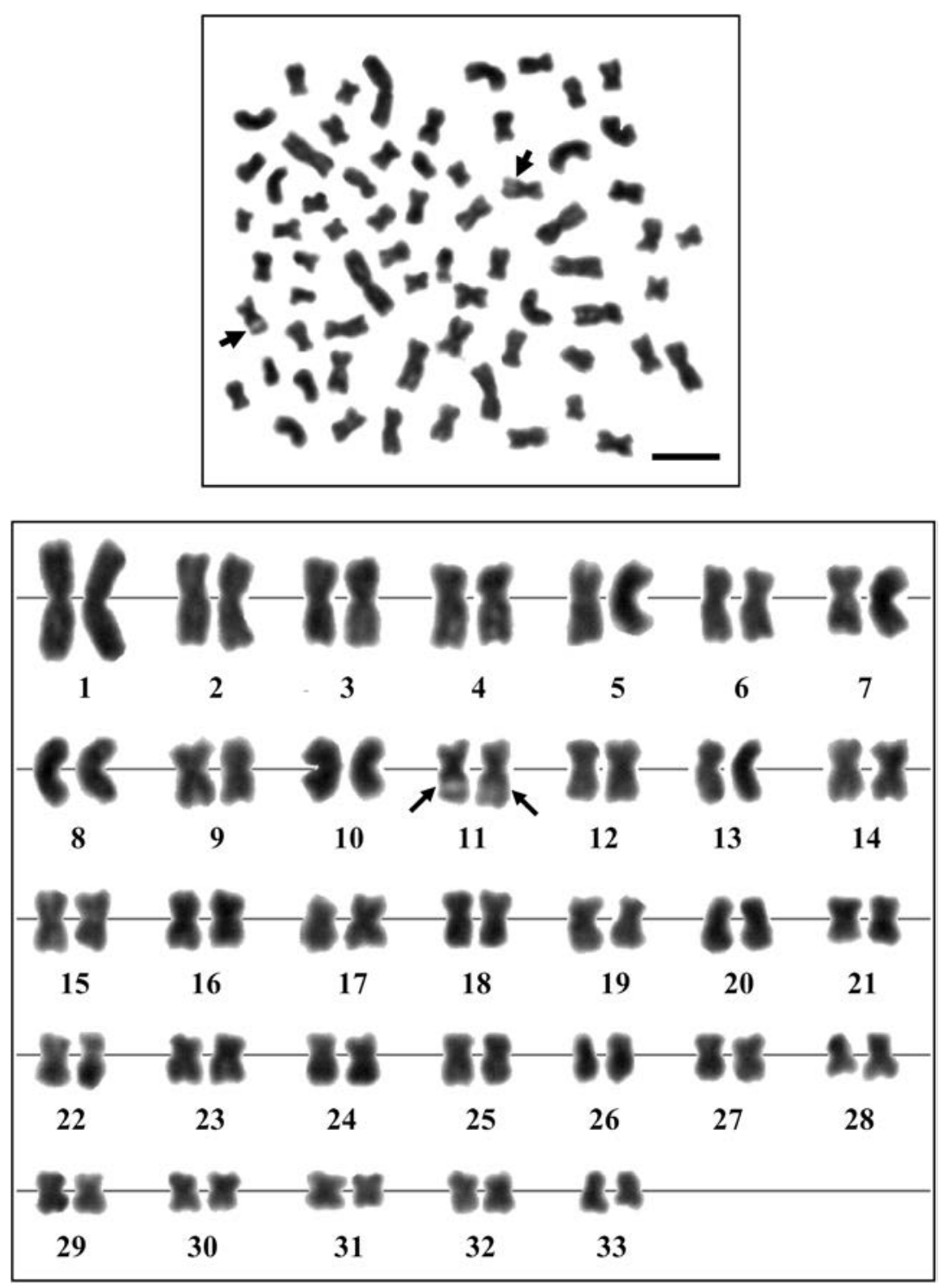

Fig. 2 Metaphase chromosome plate and karyotype of Sarika resplendens (Philippi 1846), $2 n=66$ by a conventional straining technique. Arrows indicate nucleolar organizer regions (NORs) (satellite chromosomes). The scale bar indicates $=10 \mu \mathrm{m}$.

\section{Results and discussion}

For all chromosome techniques employed here, individuals of the species show very similar results. In the ovotestis cells of $S$. resplendens, the diploid chromosome set is equal to 66 . Figure 2 shows a mitotic metaphase plate and karyotype of the ovotestis tissue with 33 pairs of chromosomes, 32 pairs of which are metacentric and the remaining pair submetacentric. The fundamental number of $S$. resplendens is 132 . The karyotype consists of six large metacentric, two large submetacentric, 26 medium metacentric, and 32 small metacentric chromosomes.

Our present report of $S$. resplendens with $2 n=66$ is consistent with others in the family Ariophantidae, including Macrochlamys asamurai, Syama diadema (Dumrongrojwattana et al. 


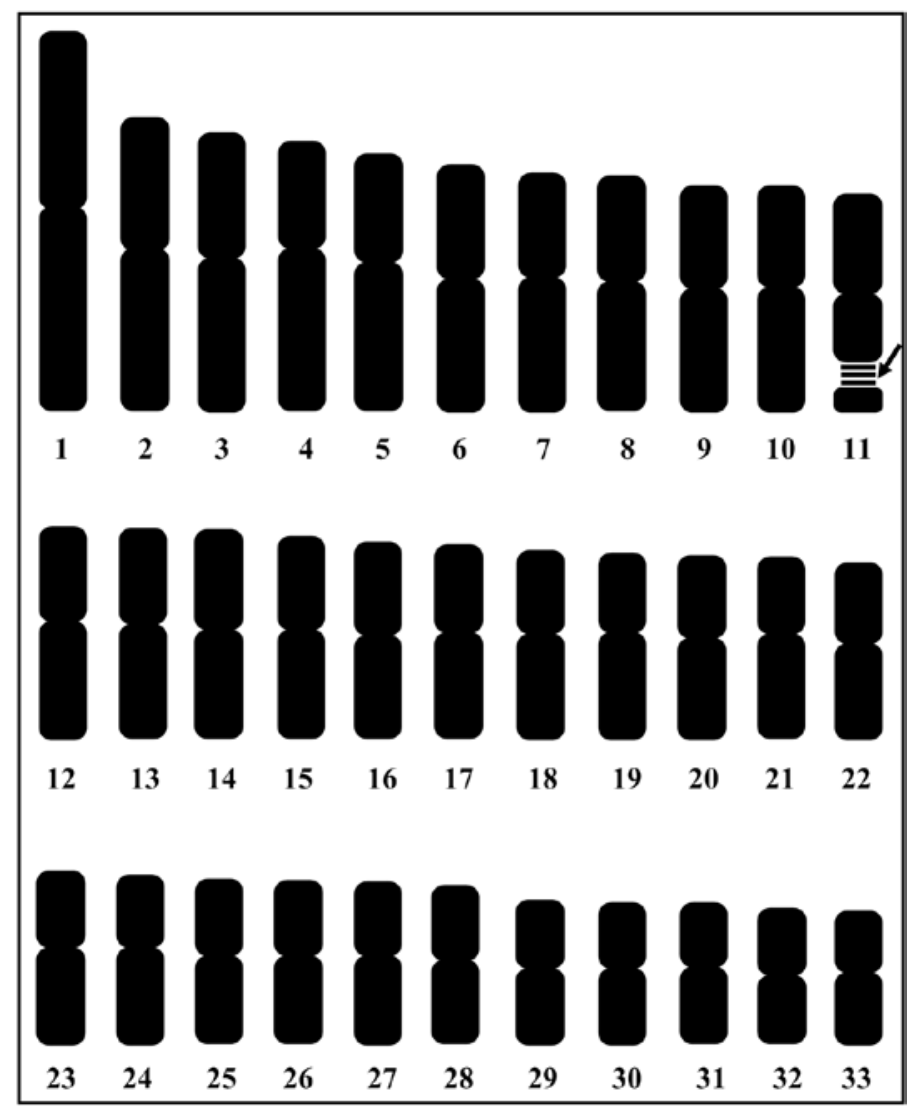

Fig. 3 Idiogram showing lengths and shapes of chromosomes of Sarika resplendens (Philippi 1846), 2n=66 by a conventional staining technique. The arrow indicates satellite chromosomes/NOR pair No. 11.

2005), Cryptozona siamensis, Hemiplecta distincta, H. weinkauffiana, Macrochlamys hepbagyla, Dyakia salangana, and Durgella libas (Dumrongrojwattana et al. 2007). However, Maneevong (2000) concluded that all Thai land snails, which were previously referred to as Macrochlamys, should be reclassified into Sarika based on anatomical characters of reproductive organs. This idea was also accepted by Sutcharit and Panha (2008) and Nabhitabhata (2009). The cytogenetic literature review related to snails by Patterson and Burch (1978) revealed that the order Stylommatophora has $2 n=10-88$. The family Anthoraccophoridae (genera Aneites and Triboniophorus) has the largest diploid chromosome number of $2 n=88$, while the family Succineidae has the smallest with $2 n=10$ 50. The family Ariophantidae has $2 n=50-64$ (Table 1).

Our present value of 132 for the NF of S. resplendens differs from the report of Mattayassook (1996) that showed the NF of C. bisgrialis, M. splendens, and Syama diadema to be 28, 32, and 40, respectively. The karyotype of $S$. resplendens comprises 64 metacentric and two submetacentric chromosomes. It is quite similar to that of $S$. asamurai, which has 58 metacentric/submetacentric chromosomes, while $S$. diadema has 56 metacentric and 10 submetacentric chromosomes (Dumrongrojwattana et al. 2005). However, the karyotype of S. resplendens differs from that of M. indica (El Alfy 1994) and S. diadema (Mattayassook 1996) (Table 1).

The present study on meiotic cell division in S. resplendens found that during metaphase I (meiosis I, reductional division), the homologous chromosomes show synapsis, which can be defined as the formation of 33 ring bivalents (32 ring bivalents of metacentric chromosomes and one ring bivalent of submetacentric chromosomes) (Fig. 4G.), and 33 haploid chromosomes at 

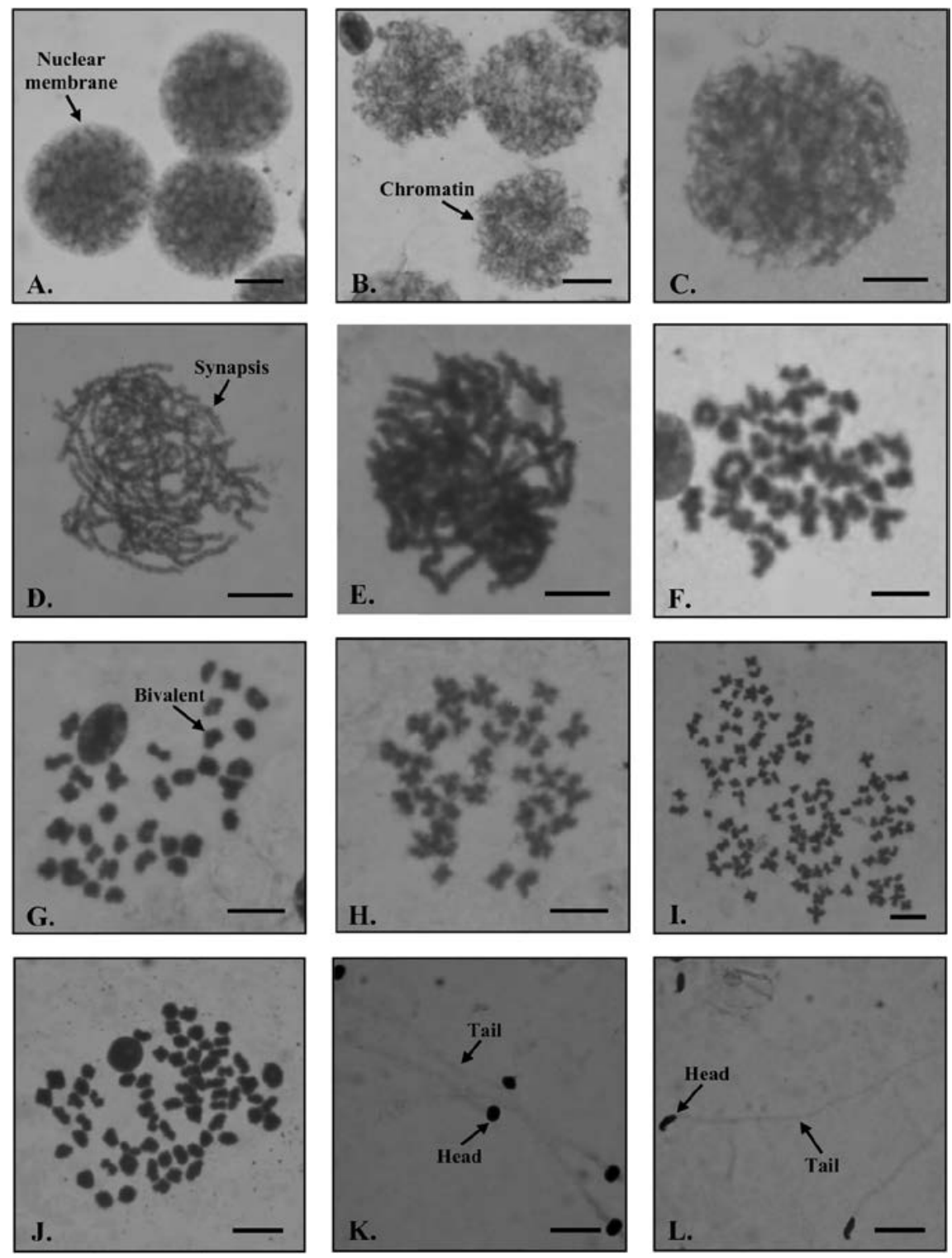

Fig. 4 Meiotic cell division of Sarika resplendens (Philippi 1846), $2 n=66$ on interphase (A.), leptotene (B.), zygotene (C.), pachytene (D.), diplotene (E.), diakinesis (F.), metaphase I (G.), metaphase II (H.), two genome doubling, $4 n$ of mitosis (I.), two genome doubling, $4 n$ of metaphase II (J.), immature spermatozoa (K.), and mature spermatozoa (L.) by a conventional staining technique. Scale bars indicate $10 \mu \mathrm{m}$.

metaphase II (meiosis II, equational division) as a diploid species (Fig. 4H.). Metacentric chromosome pair No. 1 is the largest ring bivalent, while metacentric chromosome pair No. 33 is the smallest ring bivalent. No diakinesis (prophase I) and metaphase I cells with partially paired bivalents, which are speculated to be heteromorphic sex chromosomes, and no metaphase II cells with condensed chromosomes, which are speculated to be the sex chromosomes, were detected.

In prophase I (meiosis I), we found that $S$. resplendens had distinctly observable interphase (chromatin network, Fig. 4A.), leptotene (initiation of chromosome shrinking, Fig. 4B.), zygotene (initiation of chromosome synapsis, Fig. 4C.), pachytene (completion of chromosome synapsis, 

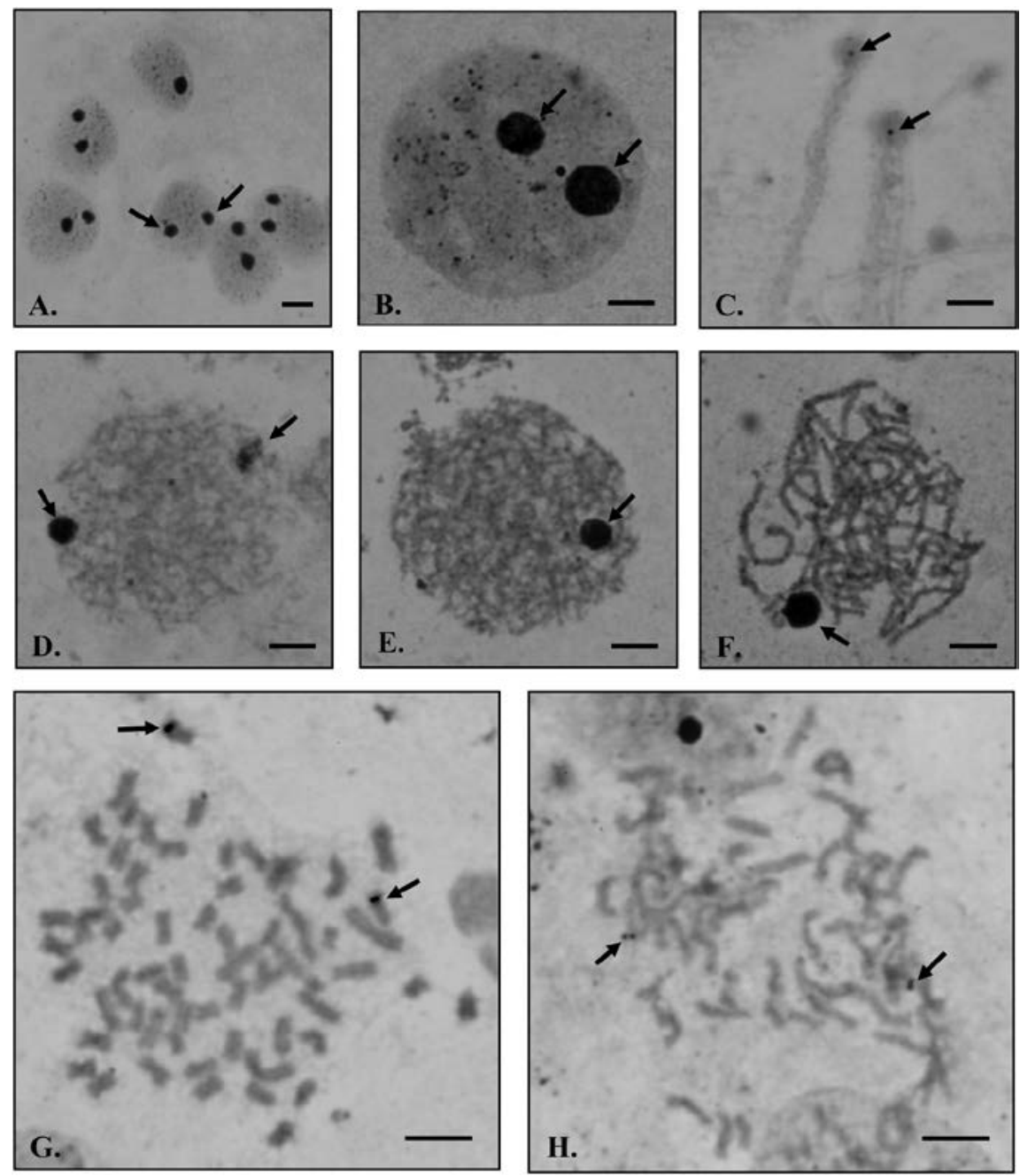

Fig. 5 Meiotic cell division of Sarika resplendens (Philippi 1846), $2 n=66$ on interphase (A and B), immature spermatozoa (C), leptotene (D), zygotene (E), pachytene (F), and mitotic cell division of metaphase chromosome plates $(\mathrm{G}$ and $\mathrm{H})$ by the Ag-NOR banding technique. Arrows indicate nucleolar organizer regions (NORs). (Scale bars indicate $=10 \mu \mathrm{m}$ ).

Fig. 4D.), diplotene (chiasma and crossing over, Fig. 4E.), and diakinesis (terminalization, Fig. 4F.). We also found that the use of colchicines caused genome doubling from $2 n$ to the two genome doubling, $4 n$ of mitosis (Fig. 4I.), and two genome doubling, $4 n$ of metaphase II (Fig. 4J.). Moreover, the round head-like immature spermatozoas (Fig. 4K.) and the hook head-like mature spermatozoas were detected (Fig. 4L.).

The results of the cytogenetic study of $S$. resplendens performed by Ag-NOR staining technique are as follows. The objective of this technique is to detect NORs, which represent the location of genes that have functions in ribosome synthesis (18S and $28 \mathrm{~S}$ ribosomal RNA). NORs produce numerous gene expressions and they are composed of more non-histone protein than other chromosome regions. Accordingly, a specific dark band (NOR-positive) is induced by the reduction of organic silver by these proteins that change silver to be dark (Sharma et al. 2002). The region adjacent to the long arm subtelomeric region of chromosome pair No. 11 showed clearly observable NORs (Figs. 5G. and 5H.). Moreover, the results gained from meiotic cell division of 
S. resplendens on interphase (Figs. 5A. and 5B.) and leptotene (Fig. 5D.) showed the two positions of NORs. In contrast, the zygotene (Fig. 5E.), pachytene (Fig. 5F.), and immature spermatozoas (Fig. 5C.) showed only one position of NOR.

Two NOR-bearing chromosomes are found in S. resplendens. In gastropods, most of the species show two NORs, whereas the Nucella lapillus (Pascoe et al. 1996) and the Fasciolaria lignaria (Vitturi et al. 2000) have a maximum number of four and eight NORs, respectively. In bivalves, 6 NOR-bearing chromosomes for karyotypes were found (Thiriot-Quievreux 2002). It has been suggested by several authors that one pair of NORs (two positions) per cell is primitive in most vertebrates (Schmid 1978) and invertebrates including molluscs (Pascoe et al. 1996, Vitturi et al. 2005).

The structure, number, and morphology of the nucleolar organizer region (NOR) may be specific to populations, species and subspecies. NOR is frequently used to compare variations, as well as to identify and explain specifications. Changes in chromosome number and structure can alter the number and structure of NOR. Robertsonian translocations may cause losses of NOR. Species,

Table 2. Mean short arm chromosome length (Ls), long arm chromosome length (Ll), total arm chromosome length (LT), relative length (RL), and centromeric index (CI) from 10 metaphases of Sarika resplendens (Philippi, 1846), $2 n=66$.

\begin{tabular}{|c|c|c|c|c|c|c|c|}
\hline $\begin{array}{l}\text { Chromosome } \\
\text { pairs }\end{array}$ & Ls & $\mathrm{Ll}$ & $\mathrm{LT}$ & CI & $\mathrm{RL}$ & $\begin{array}{l}\text { Chromosome } \\
\text { size }\end{array}$ & $\begin{array}{c}\text { Chromosome } \\
\text { type }\end{array}$ \\
\hline 1 & 0.479 & 0.549 & 1.028 & 0.534 & 0.029 & Metacentric & Large \\
\hline 2 & 0.357 & 0.435 & 0.792 & 0.548 & 0.022 & Metacentric & Large \\
\hline 3 & 0.334 & 0.412 & 0.746 & 0.552 & 0.021 & Metacentric & Large \\
\hline 4 & 0.285 & 0.435 & 0.720 & 0.605 & 0.020 & Submetacentric & Large \\
\hline 5 & 0.290 & 0.396 & 0.686 & 0.577 & 0.019 & Metacentric & Medium \\
\hline 6 & 0.305 & 0.356 & 0.661 & 0.539 & 0.018 & Metacentric & Medium \\
\hline 7 & 0.281 & 0.359 & 0.640 & 0.562 & 0.018 & Metacentric & Medium \\
\hline 8 & 0.283 & 0.345 & 0.628 & 0.549 & 0.018 & Metacentric & Medium \\
\hline 9 & 0.276 & 0.331 & 0.607 & 0.545 & 0.017 & Metacentric & Medium \\
\hline 10 & 0.271 & 0.332 & 0.603 & 0.550 & 0.017 & Metacentric & Medium \\
\hline $11^{*}$ & 0.268 & 0.314 & 0.583 & 0.539 & 0.016 & Metacentric & Medium \\
\hline 12 & 0.251 & 0.313 & 0.564 & 0.555 & 0.016 & Metacentric & Medium \\
\hline 13 & 0.257 & 0.302 & 0.559 & 0.540 & 0.016 & Metacentric & Medium \\
\hline 14 & 0.267 & 0.287 & 0.554 & 0.519 & 0.016 & Metacentric & Medium \\
\hline 15 & 0.250 & 0.289 & 0.538 & 0.536 & 0.015 & Metacentric & Medium \\
\hline 16 & 0.248 & 0.275 & 0.523 & 0.526 & 0.015 & Metacentric & Medium \\
\hline 17 & 0.234 & 0.282 & 0.516 & 0.546 & 0.014 & Metacentric & Medium \\
\hline 18 & 0.224 & 0.282 & 0.506 & 0.558 & 0.014 & Metacentric & Small \\
\hline 19 & 0.212 & 0.286 & 0.498 & 0.574 & 0.014 & Metacentric & Small \\
\hline 20 & 0.218 & 0.271 & 0.489 & 0.554 & 0.014 & Metacentric & Small \\
\hline 21 & 0.204 & 0.277 & 0.481 & 0.576 & 0.013 & Metacentric & Small \\
\hline 22 & 0.213 & 0.255 & 0.468 & 0.544 & 0.013 & Metacentric & Small \\
\hline 23 & 0.205 & 0.258 & 0.464 & 0.557 & 0.013 & Metacentric & Small \\
\hline 24 & 0.191 & 0.259 & 0.450 & 0.575 & 0.013 & Metacentric & Small \\
\hline 25 & 0.204 & 0.234 & 0.438 & 0.534 & 0.012 & Metacentric & Small \\
\hline 26 & 0.201 & 0.232 & 0.433 & 0.536 & 0.012 & Metacentric & Small \\
\hline 27 & 0.194 & 0.236 & 0.430 & 0.548 & 0.012 & Metacentric & Small \\
\hline 28 & 0.200 & 0.221 & 0.421 & 0.525 & 0.012 & Metacentric & Small \\
\hline 29 & 0.182 & 0.203 & 0.386 & 0.527 & 0.011 & Metacentric & Small \\
\hline 30 & 0.174 & 0.202 & 0.376 & 0.536 & 0.011 & Metacentric & Small \\
\hline 31 & 0.170 & 0.202 & 0.372 & 0.543 & 0.010 & Metacentric & Small \\
\hline 32 & 0.178 & 0.180 & 0.358 & 0.503 & 0.010 & Metacentric & Small \\
\hline 33 & 0.163 & 0.191 & 0.354 & 0.540 & 0.010 & Metacentric & Small \\
\hline
\end{tabular}


which have limited gene exchange due to geographical isolation, have elevated karyotype and NOR variety. Therefore, different karyotypes are found even in small, but isolated populations of these species. The use of NORs in explaining kinships, to a large extent, depends on the uniformity of this characteristic and on the degree of variety within a taxon (Yüksel and Gaffaroğlu 2008).

The important chromosome marker of $S$. resplendens is the symmetrical karyotype that was found in metacentric and submetacentric chromosomes. The largest and smallest chromosomes show size differences (approximately three folds). The chromosome markers are chromosome pair No. 1, which is the largest metacentric chromosome, and chromosome pair No. 33, which is the smallest metacentric chromosome. The chromosome length in centimeters of 10 cells in mitotic metaphase was measured. The mean short arm chromosome length (Ls), long arm chromosome length (Ll), total arm chromosome length (LT), relative length (RL), centromeric index (CI), size, and type of chromosome are presented in Table 2. An idiogram by a conventional staining technique is shown in Fig. 3. The idiogram shows the gradually decreasing length of the chromosomes. The karyotype formula of $S$. resplendens is as follows: $(2 n=66): \mathrm{L}_{6}^{\mathrm{m}}+\mathrm{L}_{2}^{\mathrm{sm}}+\mathrm{M}_{26}^{\mathrm{m}}+\mathrm{S}_{32}^{\mathrm{m}}$.

\section{Acknowledgments}

This work was financially supported by the Applied Taxonomic Research Center (ATRC) and Khon Kaen University grant; ATRC-R5304. We are indebted to Mr. Kitti Tanmueangpak and Miss Chamaiporn Worachak who studied specimens and assisted with fieldwork. Special thanks to Dr. Jolyon Dodgson for kindly checking the manuscript.

\section{References}

Blanford, W. T., and Austen, G. H. H. 1908. Mollusca, Testacellidae and Zonitidae. The fauna of British India, Taylor and Francis, London.

Chaiyasut, K. 1989. Cytogenetics and cytotaxonomy of the family Zephyranthes. Department of Botany, Faculty of Science, Chulalongkorn University, Bangkok.

Dumrongrojwattana, P., Matchacheep, S., and Yenjai, N. 2005. Karyotype of Thai Crown snails Macrochlamys asamurai Panha, 1996 and Syama diadema (Dall, 1897) (Pulmonata: Ariophantidae). Kasetsart J. (Nat. Sci.) 39: 236-241.

Dumrongrojwattana, P., Mesukkho, C., and Khunsook, C. 2007. Chromosome study of fourteen land pulmonate snails in Thailand (Pulmonata: Achatinidae, Succineidae, Helicarionidae, Ariophantidae). Khon Kaen University Research Journal 12: 102-109.

El Alfy, N. Z. 1994. Karyotype, meiosis and sperm formation in the land snail, Macrochlamys indica. Qatar University Science Journal 14: 122-128.

Hemmen, J., and Hemmen, C. 2001. Aktualisierte liste der terresrischen Gastropoden Thailands. Schriften zur Malakozoologie 18: 35-70.

Howell, W. M., and Black, D. A. 1980. Controlled silver-staining of nucleolus organizer regions with a protective colloidal developer: a 1-step method. Experientia 36: 1014-1015.

Kawano, T., and Leme, J. L. M. 1994. Chromosomes of three species of Megalobulimidae (Gastropoda: Mesurethra: Megalobulimidae) from Brazil. Malacological Review 27: 47-52.

Maneevong, A. 2000. Taxonomic revision of terrestrial snails genera Macrochlamys, Cryptozona and Hemiplecta in Thailand. M.Sc. thesis, Chulalongkorn University, Bangkok.

Mattayassook, N. 1996. Karyotype of some land pulmonate snails in Khao Ang Rue Nai and Khao Soi Dao wildlife sanctuaries. M.Sc. thesis, Chulalongkorn University, Bangkok.

Nabhitabhata, J. 2009. Checklist of Mollusca fauna in Thailand. Office of natural resources and environmaental policy and planning, Bangkok. p. 576.

Panha, S. 1996. A checklist and classification of the pulmonate snail of Thailand. Walkerana 19: 31-40.

Pascoe, P. L., Patton, S. J., Critcher, R., and Dixon, D. R. 1996. Robertsonian polymorphism in the marine gastropod Nucella lapillus: advance in karyology using r DNA loci and NORs. Chromosoma 104: 455-460.

Patterson, C. M., and Burch, J. B. 1978. Chromosome of pulmonate mollusks. In: Fretter, V., and Peak, J. (eds.). Pulmonate, Vol. 2. Academic Press, New York. pp. 171-217.

Sharma, O. P., Tripathi, N. K., and Sharma, K. K. 2002. A review of chromosome banding in fishes. In: Sobti, R. C., Obe, G., and Athwal, R. S., (eds.). Some Aspects of Chromosome Structure and Functions. Narosa Publishing House, 
New Delhi. pp. 109-122.

Schmid, M. 1978. Chromosome banding in Amphibia. I. Constitutive heterochromatin and nucleolus organizer regions in Bufo and Hyla. Chromosome 66: 361-388.

Solem, A. 1984. A world model of land snail diversity and abundance. In: Solem, A. and van Bruggen, A. C., (eds.). WorldWide Snails: Biogeographical Studies on Non-Marine Mollusca. Leiden, Brill. pp. 6-22.

Sumner, A. T. 1990. Chromosome banding. Unwin Hyman, London.

Sutcharit, C., and Panha, S. 2008. Taxonomic Re-evaluation of Sarika diadema (Dall, 1897) and S. asamurai (Panha, 1996) two endemic landsnail from Thailand (Pulmonata: Ariophantidae: Macrochlamydidae). The Raffle Bulletin of Zoology 56: 95-100.

Thiriot-Quievreux, C. 2002. Review of the literature on bivalve cytogenetics in the last ten years. Cah. Biol. Mar. 43: 1726.

Vaught, K. C. 1989. A classification of the living Mollusca. American Malacologists, Inc., Melbourne. p. 189.

Vitturi, R., Colomba, M. S., Gianguzza, P., and Pirrone, A. M. 2000. Chromosomal location of ribosomal DNA (rDNA), $(\mathrm{GATA})_{n}$ and (TTAGGG) ${ }_{n}$ telomeric repeats in the neogastropod Fasciolaria lignaria (Mollusca: Prosobranchia). Genetica 108: 253-257.

Vitturi, R., Libertini, A., Panozzo, M., and Mezzapelle, G. 1995. Karyotype analysis and genome size in three Mediterranean species of periwinkles (Prosobranchia: Mesogastropoda). Malacologia 37: 123-132.

Vitturi, R., Libertini, A., Sineo, L., Sparacio, I., Lannino, A., Gregorini, A., and Colomba, M. 2005. Cytogenetics of the land snails Cantareus asperses and C. mazzullii (Mollusca: Gastropoda: Pulmonata). Micron 36: 351-357.

Wade, C. V. M., Mordan, P. B., and Clarke, B. 2001. A phylogeny of the land snails (Gastropoda: Pulmonata). Proc. R. Soc. Lond. B268: 413-422.

Yüksel, E., and Gaffaroğlu, M. 2008. The analysis of nucleolar organizer regions in Chalcalburnus mossulensis (Pisces: Cyprinidae). J. Fish. Sci. com. 2: 587-591. 